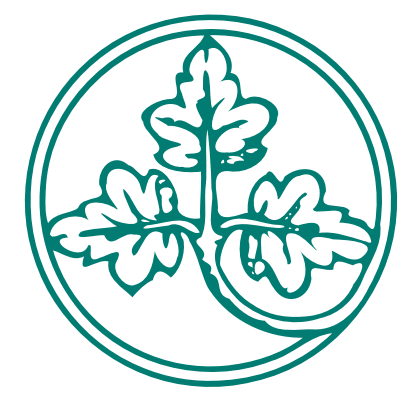

Reveal it or conceal it: On the value of second opinions in a low-entry-barriers credence goods market

Parampreet Christopher Bindra Rudolf Kerschbamer Daniel Neururer Matthias Sutter 


\section{Reveal it or conceal it: On the value of second opinions in a low-entry-barriers credence goods market}

Parampreet Christopher Bindra / Rudolf Kerschbamer / Daniel Neururer / Matthias Sutter

May 2020 


\title{
Reveal it or conceal it: On the value of second opinions in a low-entry-barriers credence goods market ${ }^{\star}$
}

\author{
Parampreet Christopher Bindra ${ }^{+}$, Rudolf Kerschbamer ${ }^{+}$, \\ Daniel Neururer ${ }^{+}$, and Matthias Sutter"
}

\begin{abstract}
Credence goods markets with their asymmetric information between buyers and sellers are prone to large inefficiencies. In theory, poorly informed consumers can protect themselves from maltreatment through sellers by asking for second opinions from other sellers. Yet, empirical evidence whether this is a successful strategy is scarce. Here we present a natural field experiment in the market for computer repairs. We find that revealing a second opinion from another expert to the seller does neither increase the rate of successful repairs nor decrease the average repair price. We assess under which conditions gathering a second opinion can be valuable.
\end{abstract}

JEL: C93, D82

Keywords: Credence goods, expert services, second opinions, natural field experiment

* We thank Loukas Balafoutas, Brit Grosskopf, Marie-Claire Villeval and seminar participants at the Uni-versities of Exeter, Linz and Innsbruck as well as conference and workshop participants at the SFB Winter School, Experimental-Day Innsbruck-Vienna, EUREGIO Workshop Trento, SMYE Brussels, IMEBESS Utrecht, AFE Chicago, ESA-NA Meeting Los Angeles for helpful comments and suggestions. Financial support from the Austrian Science Fund (SFB F63), the University of Innsbruck (Förderstipendium, Bindra) and the Deutsche Forschungsgemeinschaft (DFG, German Research Foundation) under Germany's Excellence Strategy - EXC $2126 / 1-390838866$ is gratefully acknowledged.is gratefully acknowledged.

+ Department of Economics, University of Innsbruck, Universitätsstrasse 15, 6020 Innsbruck, Austria. E-mail: parampreet.bindra@uibk.ac.at (Bindra), rudolf.kerschbamer@uibk.ac.at (Kerschbamer), daniel.neururer@uibk.ac.at (Neururer).

\# Max Planck Institute for Research into Collective Goods, Bonn, Germany, University of Cologne, Eco-nomics: Design and Behavior, Cologne, Germany; Department of Public Finance, University of Innsbruck, Innsbruck, Austria. E-mail: matthias.sutter@coll.mpg.de. 


\section{Introduction}

The uneasy feeling after blindly following the recommendations of an expert is well-known to many consumers in markets for credence goods. One possibility to avoid this feeling is to consult another expert for a second opinion. While in theory this could work as a disciplining device on experts by containing their room for exploiting less informed consumers Pesendorfer and Wolinsky, 2003), the empirical evidence indicating that gathering second opinions is a valuable strategy in credence goods markets is scarce. In fact, so far there is no study that examines the value of second opinions in credence goods markets where notable effort or competence is needed to diagnose the consumer's needs properly. In this paper, we present a natural field experiment in a credence goods market that allows us assessing the effects of gathering second opinions.

Classical examples of credence goods markets are medical treatments, education and legal services. ${ }^{1}$ These are examples of high-entry-barriers credence goods markets in the sense that expert providers have to exhibit a specific education or qualification in order to operate. By contrast, many repair services or taxi rides are examples of low-entry-barriers credence goods markets because almost everyone can start to operate in these branches without any, or very little, specific education or qualification. ${ }^{2}$

Both, low- and high-entry-barriers credence goods markets are characterized by an informational asymmetry between the expert seller and the buyer regarding the fit between the characteristics of the good or service and the needs of the buyer. In addition, the buyer is often not even ex post able to judge which quality of the good he actually received. ${ }^{3}$ As a consequence, customers in credence goods markets face the permanent risk of receiving the wrong quality (underprovision - the customer receives a lower quality than actually needed; or overprovision - the customer gets a higher quality than actually needed) or paying for more than was actually received (overcharging - the customer is charged for a more expensive service than the one that was provided).

Besides intentional mistreatment (under- or overprovision) and overcharging, a lack of effort at the diagnosis stage and incompetence on the expert's side are two important forces potentially leading to large inefficiencies in markets for credence goods (see Pesendorfer and Wolinsky, 2003; Dulleck and Kerschbamer, 2009; Schneider, 2012; Das et al., 2016; Agarwal et al., 2019). Although the underlying source of the problem is different - a moral hazard problem in the case of insufficient effort and an adverse selection problem in the case of incompetence

1 The seminal paper on credence goods is by Darby and Karni (1973). Dulleck and Kerschbamer (2006) present a unifying model of credence goods and a small set of organizing assumptions with the property that the price mechanism solves the fraudulent expert problem if those assumptions are satisfied and that most existing results on inefficiencies and fraud in credence goods markets can be reproduced by dropping one of those assumptions. By doing so the authors summarize much of the older literature on credence goods. More recent surveys are provided by Kerschbamer and Sutter (2017) and Balafoutas and Kerschbamer (2020).

2 Given that the local legislation is not asking for a specific qualification, as it is sometimes the case; e.g. taxi drivers in London have to pass a challenging test called "the knowledge" (see https://tfl.gov.uk/infofor/taxis-and-private-hire/licensing/learn-the-knowledge-of-london, accessed on 25 February 2020).

3 Other types of goods according to Nelson (1970) are ordinary goods, which have known properties; search goods, for which the properties are discovered before buying; and experience goods, for which the properties are discovered after purchasing - see Huck et al. $(2012,2016)$ who study the provision of experience goods. 
- the outcome for the consumer is similar: if the resulting misdiagnosis leads to underprovision then there is a cost for the consumer that exceeds the benefit; and if it leads to overprovision then the consumer receives a benefit that could have been obtained at a lower cost. The problems of insufficient effort at the diagnosis stage and incompetence are expected to be especially severe in low-entry-barriers credence goods markets where an adequate qualification is not enforced before market entry and hence providers' diagnostic abilities are arguably quite heterogeneous (see e.g. Schneider and Bizer, 2017; Liu et al., 2019).

In the theoretical models of Pesendorfer and Wolinsky (2003), Schneider and Bizer (2017) and Agarwal et al. (2019) an important mechanism that induces experts to invest effort at the diagnosis stage or to delegate the job to a more skilled worker at a higher cost is consumers' search for multiple opinions: If experts expect that wrong recommendations or inflated price quotes are frequently rejected, then the consumers' search for multiple opinions acts as a disciplining device, inducing experts to exert costly effort or to delegate the job to a more competent employee.

Motivated by this theoretical work on the disciplining force of consumers' search for multiple opinions, we conduct a natural field experiment in the German market for computer repairs which represents a prime example of a low-entry-barriers credence goods market.

In a first wave of our experiment we collected data for a BASELINE condition in which no second opinions were involved. Rather, mystery shoppers brought manipulated test computers to repair shops asking for a repair. Based on the most frequent outcomes in BASELINE we then decided for two treatments in which mystery shoppers mentioned a second opinion. In REVEALED-1 our mystery shoppers informed the expert that another expert had already seen the computer and that she had come to the conclusion that the device has a hardware problem which is a fairly imprecise but correct diagnosis. In REVEALED-2 the mystery shoppers informed the expert that another expert had come to the conclusion that the device is irreparable - which is a wrong diagnosis. ${ }^{4}$

We find that the relative frequency of successful repairs is only around $75 \%$ in BASELINE. This shows that we were successful in implementing a defect that is nontrivial to detect, but requires effort. In both REVEALED treatments, it turns out that revealing a second opinion to an expert does neither increase the rate of successful repairs nor decrease the average repair price conditional on the repair being successful.

These results lead us, finally, to compare different strategies of consumers and their potential effects on expected costs of a repair. We discuss three strategies. Visiting strategy 1 reflects the BASELINE treatment by simply visiting one expert and following her recommendation. Visiting strategy 2 mimics the REVEALED treatments by assuming that a consumer mentions a second opinion, but then follows the expert's recommendation. Visiting strategy 3 is based on a simulation (using data from BASELINE) and assumes to visit two different experts without informing any of them about the second opinion, and then to decide for the cheaper expert.

$4 \quad$ Note, that the BASELINE data was collected before the REVEALED data and the same computers were involved in all treatments. Given that "a hardware problem" and "irreparable" were among the most common BASELINE diagnoses, there is no deception involved in the scripts used in REVEALED. 
We can show that this strategy is the most promising one for consumers, while revealing second opinions is even worse than not gathering any second opinion at all.

The rest of the paper is organized as follows: Section 2 discusses briefly related literature. Section 3 presents the experimental design. Section 4 shows the results, and section 5 concludes the paper.

\section{Relation to previous literature}

The present study relates to two strands of previous work - the literature on second opinions and the literature on natural field experiments in credence goods markets. The pioneering theoretical work on the disciplining force of the second opinions mechanism is by Pesendorfer and Wolinsky (2003). In their model experts have to exert costly effort to determine the problem of the customer. Since the expert's diagnosis effort is unobservable and the final success of service is not contractible experts have no direct monetary incentive to exert effort at the diagnosis stage. This changes if consumers search for two matching recommendations: Since there are infinitely many problems and infinitely many treatments, two unguided guesses never yield the same outcome and given that consumers search for two matching recommendations an unguided guess never yields acceptance. In this framework, by exerting costly diagnosis effort an expert increases the probability to make a deal.

The credence good problem addressed and the mechanism at work in Schneider and Bizer (2017) and Agarwal et al. (2019) are similar to the ones in Pesendorfer and Wolinsky (2003). In another set of papers - by Wolinsky (1993), Sülzle and Wambach (2005), Dulleck and Kerschbamer (2006) and Mimra et al. (2016) - the focus is slightly different. In these studies experts learn the needs of the customer without exerting costly effort and the question addressed is whether the search for second opinions decreases the frequency of overcharging or overprovision.

Turning to field experiments in credence goods markets, most of the existing work is intentionally designed in such a way that it is easy and cheap for experts to diagnose the problem correctly, without the need to invest noteworthy effort. ${ }^{5}$ This is done because the main focus of large parts of previous field work is on intentional misbehavior on the provision and charging stage - as, e.g., in Balafoutas et al. (2013), Kerschbamer et al. (2016), Balafoutas et al. (2017), Kerschbamer et al. (2019), or Gottschalk et al. (2020). While it is important to understand the drivers of intentional mistreatment and mischarging, and ways to decrease the probability to get defrauded in such markets, the question how to induce experts to perform a serious diagnosis is obviously important as well. To the best of our knowledge, no field research has been

5 An exception is Schneider (2012) who studied whether reputational concerns matter for the diagnosis efforts and charges of car mechanics. While his paper does not concern second opinions, it took quite some effort for mechanics to find all (pre-manipulated) defects, and many did not succeed. 
conducted up to now that examines the effects of second opinions on experts' provision behavior in a context where noteworthy effort or competence in the diagnosing stage is needed to identify the needs of the customer. ${ }^{6}$

\section{Design and Procedure}

For our natural field experiment, seven mystery shoppers visited a total of 103 computer repair shops in Berlin, Bonn, Cologne and Munich (all Germany) and handed in manipulated test computers for a repair. In each city the shops were randomly selected and randomly assigned to (i) the treatments and (ii) the mystery shoppers. ${ }^{7}$ All repairs were purchased during normal shop opening times and every repair shop was visited only once. To avoid any undesired gender or age effects we hired mystery shoppers that were all in their mid to end 20ies, students, locals, and white males.

Every mystery shopper was equipped with the same model of a high class, completely refurbished desktop computer. ${ }^{8}$ When handing in at the repair shop each computer was in a perfect condition besides our manipulation. For the manipulation - which is identical among all shopvisits - we generated an excess voltage in two $8 \mathrm{~GB}$ random access memory (RAM) modules by using so-called piezo-igniters. ${ }^{9}$ As a result, the modules were defect and the computer was unable to boot. However, once the computer was switched on, a unique diagnostics beep code occurred that required additional effort to diagnose the problem correctly. ${ }^{10,11}$ Regarding repair costs we made sure that repairing the computer is a better strategy than replacing the whole machine. Specifically, with our manipulation the ex-ante estimated repair costs amount to

This is also true for the field study on dental care by Gottschalk et al. (2020). In this study the authors use standard problems which do not require particular diagnostic effort or skill to examine the effects of different factors on the extent of fraud by professional dentists. In one condition, the patient indicates to the dentist that he has uploaded his dental x-ray to an internet platform where dentists offer free advice, and that he is awaiting a response. The authors find no treatment difference in this respect, with overtreatment rates decreasing only slightly and insignificantly in this condition.

7 Due to the geographical closeness of Bonn and Cologne, shops from both cities have been clustered together first, and then randomly assigned to the mystery shoppers.

8 See the appendix for the detailed computer specifications. In total we bought 10 identical computers of this type.

9 Piezo-igniters can be found in various camping gadgets, or in lighters and the high voltage they produce by pressing a button destroys the RAM modules.

10 On the surface it might appear that the experimental designs in Kerschbamer et al. (2016) and Kerschbamer et al. (2019) are very similar to the one employed here: All these papers work with a manipulation that involves the RAM modules. However, there are subtle differences in the manipulation: In Kerschbamer et al. (2016) only one of the two RAM modules is destroyed and this leads to an easily interpretable error message that appears on the screen. In Kerschbamer et al. (2019) both RAM modules are loosened, which makes the diagnosis easy as the loosened RAM modules catch the eye of the expert immediately when she opens the computer. In the present study both RAM modules are destroyed. This is much harder to detect as it is not visible when the computer is opened and the error message is ambiguous. As a consequence of these subtle differences in the manipulation, there are large differences in the results: While the frequency of successful repairs is about $95 \%$ in Kerschbamer et al. (2016) and about $98 \%$ in Kerschbamer et al. (2019), it is only about $75 \%$ in the present study. Of course, our paper is also completely different since Kerschbamer et al. $(2016,2019)$ do not study consider second opinions.

11 The meaning of the audible diagnostic indicator can be figured out by accessing the manufacturer's website, or other (online) sources. Hinting towards mechanics' awareness of this diagnostics system, some repair shops mentioned the diagnostics beep code and its meaning on their invoice. For the diagnostics beep codes see e.g. https:/www.dell.com/support/article/at/de/atbsdt1/sln293445/understanding-beep-codeson-a-dell-desktop-pc?lang=en (accessed on 25 February 2020). 
about 200 euro while the replacement price of the computer (not new, but in the same refurbished quality) was about 750 euro at the time of the study. ${ }^{12}$

We first collected data for our BASELINE treatment. In this treatment, the mystery shopper entered the repair shop, saying "I bought this computer used, and it does not start." and asked for a repair. We instructed our mystery shoppers to leave the shop before the expert stated her diagnosis, in order to provide sufficient moral wiggle room for the expert. Moreover, our helpers were instructed to stick to the script and we provided them pre-formulated and standardized answers for possible questions from the expert. Usually the shop called the helper after a few days with a first diagnosis and the estimated cost of the repair. In order to compare our results, we instructed our mystery shoppers to accept all repair offers up to an amount of 500 euro, and to contact us in case the repair proposal exceeded this threshold, which did not happen.

Based on the results and the diagnoses from our BASELINE treatment (reported in Subsection 4.1) we decided to have two treatments in which the mystery shopper revealed to have acquired a second opinion. In both, the general procedure is exactly the same as in BASELINE, except for a slightly modified script when handing in the computer: In REVEALED-1 the mystery shoppers added to the script of the BASELINE treatment the phrase: "Another computer shop has already seen the computer and diagnosed a problem with the hardware. I would rather get a second opinion and that's why I'm here.". In REVEALED-2 the mystery shopper added to the script of the BASELINE treatment the phrase: "Another computer shop has already seen the computer and diagnosed a problem with the hardware which is irreparable. I would rather get a second opinion and that's why I'm here.".

After picking up the computer from the shops, our helpers checked whether it worked. For the remainder of this paper we define the rate of successful repairs as the relative frequency of cases where the computer worked properly when we picked it up.

\section{Results}

We collected a total of 103 observations from different repair shops; 33 observations for the BASELINE treatment, 35 observations for REVEALED-1, and 35 observations for REVEALED-2. Out of these 103 observations, we exclude two shops (one in each of our REVEALED treatments) from our data base, as those shops first accepted our computers for repair, but then stated in the first diagnosis that they are not fixing any hardware problems. All other shops inclusive those which accepted our computer for repair but stated after a few days that it is impossible to repair it for whatever reasons - are included in the following analysis.

12 After conducting an intense online search, we estimated the average price for one 8 GB RAM module to be 78 euro plus the average diagnosis cost of 37.90 euro. As two RAM modules have been built into each computer, this sums up to a 'fair' repair price 193.90 euro. This price is comparable to the mean repair price we find in our BASELINE treatment. The replacement value corresponds to the average price we paid for one computer in a refurbished condition because we purchased the computers in two waves at slightly different prices. 


\subsection{Experimental results for BASELINE and REVEALED}

Figure 1 shows the relative frequencies of a successful repair, conditional on the experimental treatment. From the 33 shops in our BASELINE treatment, 25 (75.76\%) managed to repair the computer. Four shops (12.12\%) claimed that the computer is irreparable und recommended to replace it. The remaining four shops (12.12\%) recommended to the undercover shopper to visit another shop for a second opinion. A successful repair rate of only $75 \%$ is very low in comparison to other computer repair studies (Kerschbamer et al., 2016, 2019) with rates above $95 \%$, showing that our manipulation required an expert's effort to solve the problem.

In REVEALED-1, $67.65 \%$ of the 34 shops repaired the computer successfully, and the corresponding rate is $79.41 \%$ for the 34 shops in REVEALED-2. Pooling REVEALED- 1 and REVEALED2 to one REVEALED category for the treatments with a second opinion yields a rate of successful repairs of $73.53 \%$. None of the comparisons to BASELINE are significant, neither when pooling both REVEALED-treatments nor when considering them separately (Fisher's exact tests; $p>0.10$ for all comparisons). This implies that we have to reject the hypothesis according to which mentioning that another expert has already made a diagnosis leads to a higher rate of successful repairs as a consequence of exerting more effort.

Figure 2 shows the average repair price in case of a successful repair for the three treatments. The repair price is 188.73 euro in BASELINE, 211.73 euro in the REVEALED-1 treatment, and 242.30 euro in the REVEALED-2 treatment. For the pooled REVEALED category, the mean repair

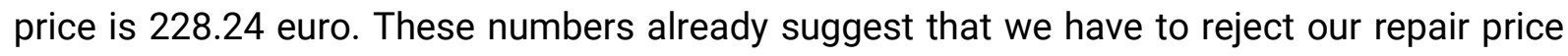
hypothesis, too, in which we had expected lower repair prices in the REVEALED-treatments. Actually, the opposite is true. Prices in REVEALED-2 are significantly larger than in BASELINE ( $p<0.05$; Mann-Whitney U-test), and pooling both REVEALED-treatments leads also to significantly higher prices in the latter treatments than in BASELINE ( $p=0.01$; Mann-Whitney U-test). We summarize the results in the experimental treatments as follows:

Result 1: Overall, the rate of successful repairs is only about $75 \%$ across all treatments. In the remaining $25 \%$ of the cases, experts are unable to diagnose the problem properly (and often recommend buying a new computer). Mentioning a second opinion does not increase the likelihood of a successful repair; nor does it reduce the charged prices. On average, prices are even $20 \%$ higher when a second opinion is mentioned. 
Figure 1: Relative frequency of successful repairs

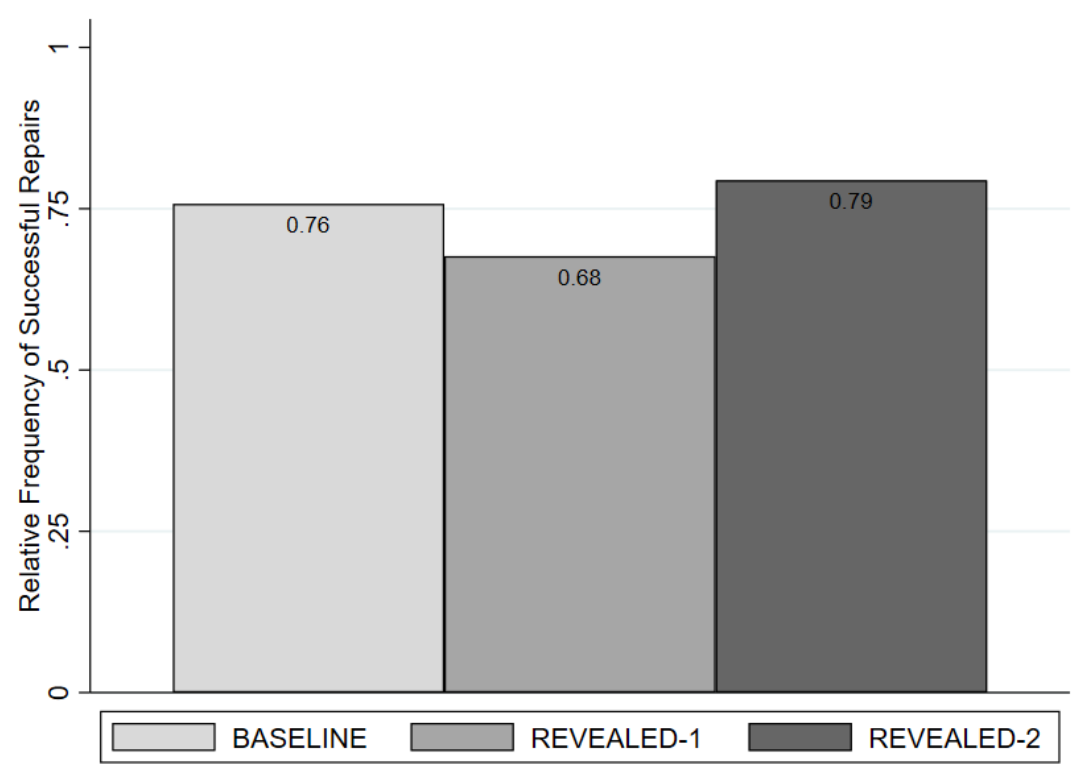

Notes: Successful repairs per treatment ( $\mathrm{N}=33$ in BASELINE, $\mathrm{N}=34$ in the REVEALED-1 treatment, and $\mathrm{N}=34$ in the REVEALED-2treatment).

Figure 2: Average repair price conditional on the repair being successful

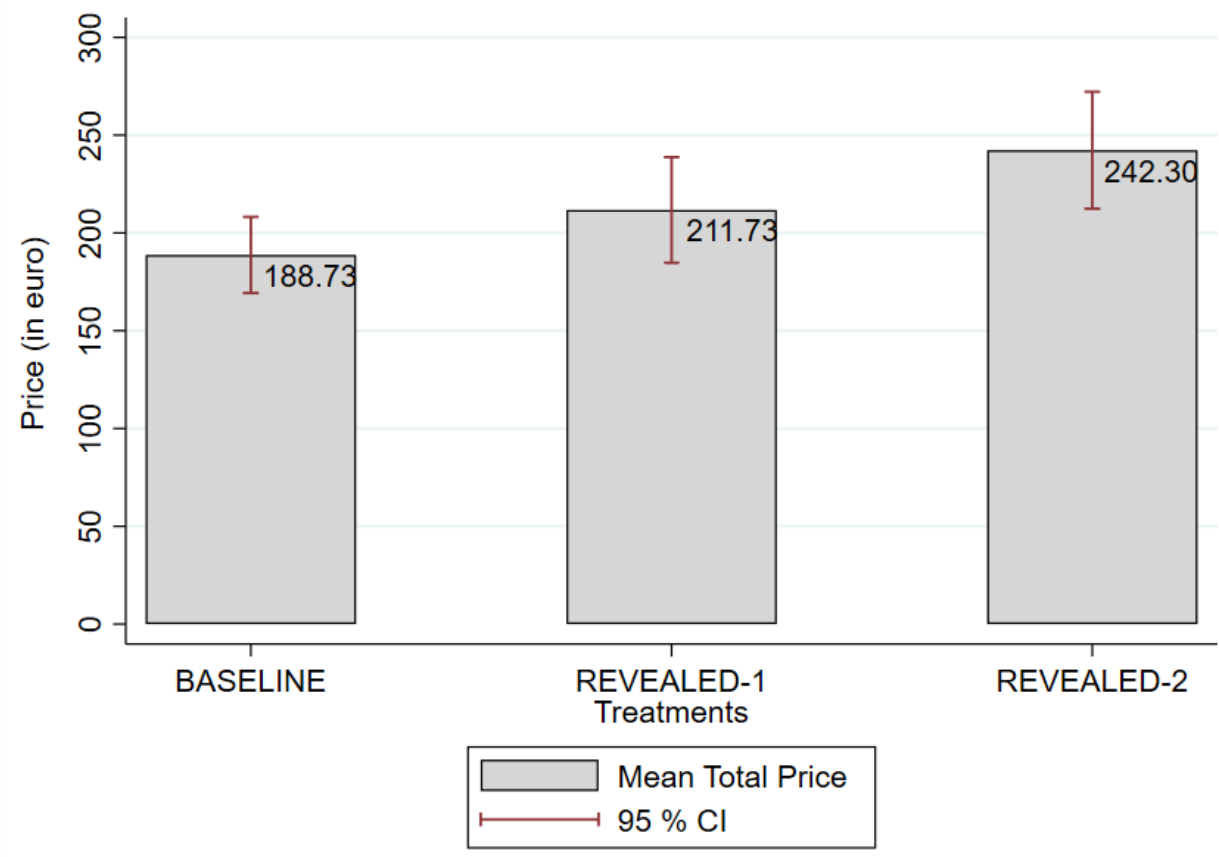

Notes: Average repair price per treatment in case of a successful repair ( $\mathrm{N}=25$ in BASELINE, 23 in the REVEALED1 treatment, and 27 in the REVEALED-2 treatment, respectively). Average price (in euro) indicated in the top right corner of each bar. Error bars, mean \pm SEM. 


\subsection{Assessing different strategies on whether or not to reveal a second opinion}

Based on our experimental findings, we can now quantify the value of different strategies with respect to revealing or concealing a second opinion. We do so in a simulation in which we take diagnosis costs into account, but ignore potential search costs. ${ }^{13}$ We compare three strategies and their expected costs for the consumer.

- Strategy 1 (S1): Visit one expert and follow her recommendation.

- Strategy 2 (S2): Visit one expert, mention that another expert has already made a diagnosis and follow the expert's recommendation.

- Strategy 3 (S3): Visit two experts without informing any of them that another opinion has been or will be obtained and decide for the cheaper expert.

For strategy S1, we can use our data from BASELINE to calculate the expected repair costs of this strategy that requires the following plan of actions: if the expert gives a price quote, then accept it; if the expert recommends replacing the computer, then replace it; and if she recommends visiting another expert, then play S1 once more. For this calculation we use the average diagnosis fee of 37.90 euro that was charged by our shops. For a new computer, we take the average costs of the computers when we bought them, i.e., 750 euro. Using the relative frequencies of successful repair $(75.76 \%)$ and recommendations for buying a new computer (12.12\%) or getting some other expert's advice $(12.12 \%)$, this yields as the expected costs of this strategy: $C_{S 1}=0.7576 \times 188.73+0.1212 \times(37.90+750)+0.1212 \times\left(37.90+C_{S 1}\right)$. Solving this expression for $C_{S 1}$ yields $C_{S 1}=276.59$ euro.

Strategy S2 suggests the following plan of actions: visit one expert and mention that another expert has already made a diagnosis; if the expert gives a price quote, then accept it; if the expert recommends replacing the computer, then replace it; and if she recommends visiting another exert, then play S2 once more. Pooling both REVEALED-treatments where customers mentions a second opinion yields $C_{S 2}=0.7353 \times 228.24+0.1912 \times(37.90+750)+0.0735 \times$ $\left(37.90+C_{s 2}\right)$. Solving this expression for $C_{s 2}$ yields $C_{s 2}=346.74$ euro, which is about $25 \%$ more expensive in expectation than strategy $\mathrm{S} 1 .{ }^{14}$

Strategy S3 asks whether gathering two opinions instead of one without informing any of the experts about the second opinion is beneficial for consumers. More precisely, this strategy implies the following: visit two experts; if both give a price quote, then decide for the cheaper one; if one expert gives a price quote and the second recommends replacing the computer or if one expert gives a price quote and the second recommends searching for a second opinion, then decide for the shop with the quote; if one shop recommends replacing the computer while the second recommends searching for a second opinion or both recommend searching for a

13 Search costs would be difficult to measure. However, since the repair shops in the four cities of our experiment are often close to each other, potential transportation costs (with respect to distance and time of travel) should be small.

14 Considering both REVEALED-treatments separately would yield expected costs for strategy S2 of 376.14 euro for REVEALED-1 and of 316.32 euro for REVEALED-2, respectively. 
second opinion, then play S3 once more; and if both experts recommend replacing the computer then replace it.

To calculate the expected cost of applying S3 we have first to calculate the expected repair price for the case where the customer receives two price quotes and decides for the lower one. To derive this figure, we draw from the 25 BASELINE observations with a price quote randomly two observations and record the cheaper one. Then we return the two observations and repeat the drawing 10,000 times. We then calculate the average of the cheaper price in the 10,000 paired quotes. This calculation yields an average of 161.88 euro. From this, we can derive the expected cost of following S3 as follows: $\mathrm{C}_{\mathrm{s} 3}=0.5740 \times(37.90+161.88)+0.1836$ $\times(37.90+188.73)+0.1836 \times(37.90+188.73)+0.0294 \times\left(37.90+37.90+C_{s 3}\right)+0.0147 \times$ $\left(37.90+37.90+C_{s 3}\right)+0.0147 \times(37.90+37.90+750)$. Solving this expression for $C_{s 3}$ yields $C_{S 3}=223.22$ euro.

While we have already seen that revealing a second opinion to an expert does neither reduce the average repair price nor increase the likelihood of a successful repair (when comparing BASELINE to the two REVEALED-treatments), our assessment of the expected costs of different consumers' strategies has confirmed that strategy S2 is more costly in expectation that strategy S1. Most importantly, however, our assessment has revealed that second opinions can help customers if they do not reveal them. Strategy S3 has considerably lower expected costs (of around 223 euro) than strategy S1 (with around 277 euro) and strategy S2 (with 347 euro). The comparison yields a large benefit for the consumer of at least 50 euro, which amounts to about $20 \%$ of the average cost of following strategy S1 without any second opinion. We therefore conclude:

Result 2: Gathering two opinions without informing any of the experts about the second opinion is profitable for the consumer: Although this course of action leads to a duplication of diagnosis costs, it is overcompensated by a decrease in the average price for a successful repair and in the probability of unnecessarily replacing the computer. Overall, by gathering (but not revealing) two opinions instead of one the consumer saves in expectation at least $20 \%$ of the repair cost.

\section{Conclusion}

The experiment reported in this paper is the first natural field experiment analyzing the effects of second opinions on experts' provision behavior in a context where noteworthy effort or competence in the diagnosing stage is needed to identify the needs of the customer. We have documented that a high rate of unsuccessful repairs (25\%) leads to pronounced inefficiencies in the low-entry-barriers credence goods market under consideration. Misdiagnoses that lead to the replacement of the computer are the major driver for these inefficiencies. The reason for this is twofold: First, the value of the computer is much higher than the average repair cost and, second, the probability that a customer ends up with the recommendation to replace the computer exceeds ten percent. Mentioning that a second opinion has previously been obtained has - in contrast to our ex ante hypothesis based on theoretical considerations - no positive impact on the rate of successful repairs. Also, the average repair price conditional on 
the repair being successful is not reduced, to say the least, when the expert is informed that another expert has already stated a diagnosis. However, getting a second opinion and processing it privately is profitable for customers. Overall, by gathering (but not revealing) two opinions instead of one the consumer saves in expectation at least $20 \%$ of the repair cost.

Overall our results suggest that in a low-entry-barriers credence goods market it might be good advice for consumers to gather, in fact, a second opinion and to process it privately - instead of feigning one or blindly accepting the first recommendation. More generally, the net benefit to the consumer of gathering multiple opinions and processing them privately depends on the heterogeneity of the behavior of experts in the market under consideration. In our case the heterogeneity in repair prices conditional on the repair being successful is not pronounced enough to justify visiting two experts - it is the reduction in the probability of following the wrong recommendation to replace the computer that makes the difference. ${ }^{15}$ Of course, more research is needed to find out whether our findings are robust in different settings and markets. Yet, given the revenues in computer repair markets are substantial (e.g., for Germany only they exceed 1.3 billion euro per year ${ }^{16}$ ), even if our results would be confined to this specific market, they would have significant implications for optimal strategies of customers to avoid exploitation and inefficiencies. Moreover, our empirical results are also important to qualify and complement the theoretical work on the value of second opinions. While it seems straightforward to assume that gathering second opinions has some disciplining power on experts, our empirical evidence has shown that there are subtle mechanisms - whether or not to reveal a second opinion to an expert - that make second opinions either valuable or actually useless.

15 The average repair price in BASELINE is 188.73 euro. The expected repair price for the case where the customer receives two price quotes and decides for the cheaper one is 161.88 euro. The difference between these two numbers is 26.85 euro - which is less than the cost of an additional diagnosis (which was on average 37.90 euro).

16 Data from a structural survey in the service sector in 2014 of the Statistisches Bundesamt; see https://www.destatis.de/EN/Themes/Economic-Sectors-Enterprises/Enterprises/ICT-Enterprises-ICT-Sector/Tables/ictb-03-enterprises-persons-employed-turnover-investments.html for details (accessed: 11 May 2020). 


\section{References}

Agarwal, R., Liu, C.-W. \& Prasad, K., 2019, Personal research, second opinions, and the diagnostic effort of experts, Journal of Economic Behavior \& Organization, 158, p. 4461.

Balafoutas, L., Beck, A., Kerschbamer, R. \& Sutter, M., 2013, What drives taxi drivers? a field experiment on fraud in a market for credence goods, Review of Economic Studies, 80(3), p. 876-891.

Balafoutas, L. \& Kerschbamer, R., 2020, Credence goods in the literature: What the past fifteen years have taught us about fraud, incentives, and the role of institutions, Journal of Behavioral and Experimental Finance, 26.

Balafoutas, L., Kerschbamer, R. \& Sutter, M., 2017, Second-degree moral hazard in a real-world credence goods market, The Economic Journal, 127(599), p. 1-18.

Darby, M. R. \& Karni, E., 1973, Free competition and the optimal amount of fraud, The Journal of law and economics, 16(1), p. 67-88.

Das, J., Holla, A., Mohpal, A. \& Muralidharan, K., 2016, Quality and accountability in health care delivery: Audit-study evidence from primary care in rural India, American Economic Review, 106(12), p. 3765-3799.

Dulleck, U. \& Kerschbamer, R., 2006, On doctors, mechanics, and computer specialists: The economics of credence goods, Journal of Economic literature, 44(1), p. 5-42.

Dulleck, U. \& Kerschbamer, R., 2009, Experts vs. discounters: Consumer free-riding and experts withholding advice in markets for credence goods. International Journal of Industrial Organization, 27(1), p. 15-23.

Gottschalk, F., Mimra, W. \& Waibel, C., 2020, Health services as credence goods: A field experiment, The Economic Journal, forthcoming.

Huck, S., Luenser, G. K. \& Tyran, J. R., 2012, Competition fosters trust. Games and Economic Behavior, 76(1), p. 195-209.

Huck, S., Luenser, G. K. \& Tyran, J. R., 2016, Price competition and reputation in markets for experience goods, An experimental study. RAND Journal of Economics 47, p. 99-117.

Kerschbamer, R., Neururer, D. \& Sutter, M., 2016, Insurance coverage of customers induces dishonesty of sellers in markets for credence goods, Proceedings of the National Academy of Sciences, 113(27), p. 7454-7458.

Kerschbamer, R., Neururer, D. \& Sutter, M., 2019, Credence goods markets and the informational value of new media: A natural field experiment, IZA Discussion Paper, No.12184. 
Kerschbamer, R. \& Sutter, M., 2017, The economics of credence goods-a survey of recent lab and field experiments, CESifo Economic Studies, 63(1), p. 1-23.

Liu F., Rasch A., Schwarz, M. A. \& Waibel, C., 2019, The role of diagnostic ability in markets for expert services, Available at SSRN: https://ssrn.com/abstract=3491607.

Mimra, W., Rasch, A. \& Waibel, C., 2016, Second opinions in markets for expert services: Experimental evidence, Journal of Economic Behavior \& Organization, 131, p. 106125.

Nelson, P., 1970, Information and consumer behavior, Journal of political economy, 78(2), p. 311-329.

Pesendorfer, W. \& Wolinsky, A., 2003, Second opinions and price competition: Inefficiency in the market for expert advice, The Review of Economic Studies, 70(2), p. 417-437.

Schneider, H. S., 2012, Agency problems and reputation in expert services: Evidence from auto repair, The Journal of Industrial Economics, 60(3), p. 406-433.

Schneider, T. \& Bizer, K., 2017, Effects of qualification in expert markets with price competition and endogenous verifiability, CEGE Discussion Papers, No. 317.

Sülzle, K. \& Wambach, A., 2005, Insurance in a market for credence goods, Journal of Risk and Insurance, 72(1), p. 159-176.

Wolinsky, A., 1993, Competition in a market for informed experts' services, The RAND Journal of Economics, p. 380-398. 


\section{Appendix}

Specification of our computers

We bought 10 identical tower computers to be able to speed up the data collection process by handing them to different shops at the same time. All of the computers were completely refurbished. The price per computer was 750 euro. In the following, we list the exact configuations of our computers:

Dell Optiplex XE2 i5 4x 2,9GHz, 2x 8GB RAM, 2x 500GB harddrive, NVIDIA Quadro NVS 510 V3, Win10 Pro 